Pacific Journal of Mathematics

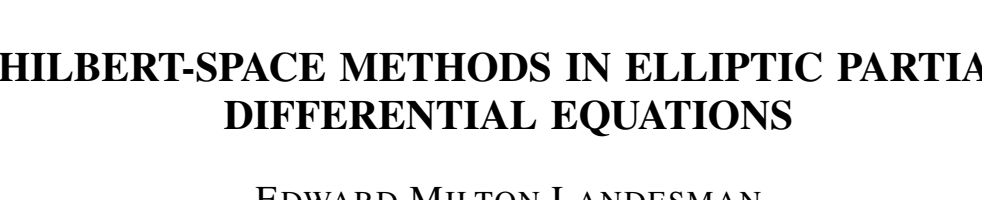




\title{
HILBERT-SPACE METHODS IN ELLIPTIC PARTIAL DIFFERENTIAL EQUATIONS
}

\author{
EDWARD M. LANDESMAN
}

The purpose of this paper is to study, together with applications, those aspects of the theory of Hilbert Space which are pertinent to the theory of elliptic partial differential equations. This involves the study of an unbounded operator $A$ from one Hilbert Space to another together with its adjoint $A^{*}$, its pseudo-inverse or generalized reciprocal $A^{-1}$, and its *-reciprocal $A^{\prime}=A^{*-1}$. In order to carry out the results, further properties of the operators $A^{-1}$ and $A^{\prime}$ are developed in this paper.

The concept of ellipticity of a partial differential operator is introduced via the properties of an operator in a suitably chosen Hilbert Space. This Hilbert Space is the one defined by the operator $G_{k}$, that is, the operator which maps a function into itself and its first $k$ derivatives. It is shown that elliptic operators are those that behave in a topological sense the same as closed and dense restrictions of the operator $G_{k}$. Several other characterizations of elliptic operators and given and their relation to each other is explained. This approach yields existence theorems for strong solutions of elliptic partial differential equations and provides methods for gaining strong solutions from weak solutions.

The $\mathscr{K}_{-k}$ spaces that arise from the so-called negative norms and that have been used effectively by several authors in the study of elliptic partial differential equations are obtained by the use of the *-reciprocal of the operator $G_{k}$.

Simple examples which illustrate the above theory are provided.

1. Preliminaries. We will mainly be interested in Hilbert Spaces. We denote our Hilbert Spaces by $\mathscr{H}$, $\mathscr{H}^{\prime}, \mathscr{H}^{\prime \prime}$, etc. When we write $\mathscr{H}$ and $\mathscr{H}^{\prime}$, they may coincide. Inner products in each Hilbert Space will be denoted as follows:

If we are concerned with an inner product in $\mathscr{H}$, and $x_{1}, x_{2} \in \mathscr{H}$, we denote their inner product in $\mathscr{H}$ by: $\left(x_{1}, x_{2}\right) \mathscr{H}$. For $x \in \mathscr{H}$, its norm will be denoted by $\|x\|_{\mathscr{C}}$. If no ambiguity is present, we will omit the subscripts $\mathscr{H}$ in both the inner product and the norm.

Suppose that $A$ is a linear transformation from $\mathscr{H}$ to $\mathscr{H}^{\prime}$. Denote the domain of $A$ by $\mathscr{D}_{A}$, the range of $A$ by $\mathscr{R}_{A}$ and the null space of $A$ by $\mathscr{N}_{A}$.

The closure of a subclass $\mathscr{B}$ of $\mathscr{H}$ will be denoted by $\overline{\mathscr{B}}$. By a closed subset of $\mathscr{H}$ will be meant one that is closed relative to strong convergence. 
The orthogonal complement of $\mathscr{B}$ in $\mathscr{H}$ will be denoted by $\mathscr{B}^{\perp}$. $\mathscr{B}^{\perp}$ is certainly a subspace of $\mathscr{H}$.

We will use the term operator to denote a closed, dense, linear transformation.

2. The pseudo-inverse of an operator, its adjoint, and related results. In this section we define the concept of the "pseudo-inverse" of an operator which is of particular interest in this paper. We then follow with some basic results which the "pseudo-inverse" shares. In order to define the pseudo-inverse of an operator, it is convenient to introduce the ordered pair definition of a linear transformation. We begin as follows:

Consider a Hilbert-Space $\mathscr{H}^{\prime}$ and a Hilbert-Space $\mathscr{H}^{\prime \prime}$ and let $\mathscr{H}=\mathscr{H}^{\prime} \times \mathscr{H}^{\prime \prime}$ be their Cartesian product. Clearly $\mathscr{H}$ is a Hilbert Space with inner product being the sum of the inner products of $\mathscr{H}^{\prime}$ and $\mathscr{H}^{\prime \prime}$. Let $\mathscr{H}_{0}^{\prime}=\mathscr{H}^{\prime} \times\{0\}, \mathscr{H}_{0}^{\prime \prime}=\{0\} \times \mathscr{H}^{\prime \prime} . \quad \mathscr{H}_{0}^{\prime}$ and $\mathscr{H}_{0}^{\prime \prime}$ are isomorphic replicas of $\mathscr{H}^{\prime}$ and $\mathscr{H}^{\prime \prime}$ respectively. Suppose $\mathscr{A}$ is a closed linear subspace of $\mathscr{H}$. Denote an element of $\mathscr{A} \subseteq \mathscr{H}$ by: $\{x, y\}$, where $x \in \mathscr{H}^{\prime}$ and $y \in \mathscr{H}^{\prime \prime}$.

If $\mathscr{A} \cap \mathscr{H}_{0}^{\prime \prime}=0$, then $\mathscr{A}$ defines a linear transformation from $\mathscr{X}^{\prime}$ into $\mathscr{L}^{\prime \prime}$. For, if $\left\{x, y_{1}\right\}$ and $\left\{x, y_{2}\right\}$ are elements of $\mathscr{A}$, then $\left\{0, y_{1}-y_{2}\right\} \in \mathscr{A}$, i.e., $y_{1}=y_{2}$. We denote this linear transformation by $A$. Furthermore, since $\mathscr{A}$ is closed, the transformation $A$ is closed.

If $\mathscr{A}^{\perp} \cap \mathscr{L}_{0}^{\prime}=0$, then $A$ is in fact dense. For, suppose not; then there exists $\left\{x_{0}, 0\right\} \in \mathscr{C}_{0}^{\prime}, x_{0} \perp \mathscr{D}_{A}, x_{0} \neq 0$. Furthermore, $\left\{x_{0}, 0\right\} \in$ $\mathscr{A}^{\perp}$. But this contradicts the fact that $\mathscr{H}^{\perp} \cap \mathscr{H}_{0}^{\prime}=0$.

In a like manner, $\mathscr{A}^{\perp}$ defines a linear transformation $-A^{*}$ from $\mathscr{H}^{\prime \prime}$ to $\mathscr{C}^{\prime}$. Summarizing, we have the following: If

$$
\mathscr{A} \cap \mathscr{L}_{0}^{\prime}=0 \text {, }
$$

then $\mathscr{A}$ defines a closed linear transformation $A$ from $\mathscr{X}^{\prime}$ to $\mathscr{C}^{\prime \prime}$. If

$$
\mathscr{A}^{\perp} \cap \mathscr{H}_{0}^{\prime}=0 \text {, }
$$

then $A$ is dense and $\mathscr{A}^{\perp}$ defines a dense linear transformation $-A^{*}$ from $\mathscr{H}^{\prime \prime}$ to $\mathscr{H}^{\prime}$.

Suppose that the relations (2.1) and (2.2) are satisfied. Let

$$
\mathscr{A}_{0}=\mathscr{A} \cap \mathscr{H}_{0}^{\prime}, \quad \mathscr{A}_{0}^{*}=\mathscr{H}^{\perp} \cap \mathscr{C}_{0}^{\prime \prime}
$$

which correspond to the null spaces of $A$ and $A^{*}$ respectively. Then

$$
\mathscr{A}=\mathscr{A}_{0}+\mathscr{C}, \quad \mathscr{A}^{\perp}=\mathscr{X}_{0}^{*}+\mathscr{C}^{*}
$$

where $\mathscr{C} \perp \mathscr{A}_{0}$ and $\mathscr{C}^{*} \perp \mathscr{A}_{0}^{*}$. Moreover, 


$$
\mathscr{H}=\mathscr{A}+\mathscr{A}^{\perp}=\left(\mathscr{A}_{0}+\mathscr{C}\right)+\left(\mathscr{A}_{0}^{*}+\mathscr{C}^{*}\right) .
$$

Observe that when $\mathscr{C}$ is decomposed as in (2.5), $\mathscr{A}_{0}+\mathscr{C}$ completely determines the operator $A$ and $\mathscr{A}_{0}^{*}+\mathscr{C}^{*}$ completely determines its adjoint $A^{*}$.

Rearranging (2.5), we have

$$
\mathscr{H}=\mathscr{A}+\mathscr{A}^{\perp}=\left(\mathscr{A}_{0}+\mathscr{C}^{*}\right)+\left(\mathscr{A}_{0}^{*}+\mathscr{C}\right) .
$$

The linear subspace $\mathscr{A}_{0}^{*}+\mathscr{C}$ defines an operator $A^{-1}$ from $\mathscr{X}^{\prime \prime}$ to $\mathscr{C}^{\prime}$ called the pseudo-inverse or generalized reciprocal of $A$. The linear subspace $\mathscr{A}_{0}+\mathscr{C}^{*}$ defines the operator $-A^{\prime}$ from $\mathscr{H}^{\prime}$ to $\mathscr{X}^{\prime \prime}$. The operator $A^{\prime}$ is the pseudo-inverse of the adjoint, $A^{*-1}$ of $A$. We call $A^{\prime}$ the *-reciprocal of $A$. We observe that $A$ and $A^{\prime}$ both take $\mathscr{H}^{\prime}$ into $\mathscr{H}^{\prime \prime}$ while $A^{*}$ and $A^{-1}$ both take $\mathscr{C}^{\prime \prime}$ into $\mathscr{C}^{\prime}$. Summarizing, we have the following:

Proposition 2.1. The domain $\mathscr{D}_{A^{-1}}$ of $A^{-1}$ is the direct sum of $\mathscr{R}_{A}$ and $\mathscr{R}_{A}^{\perp}$. The range of $A^{-1}$ is the intersection of $\mathscr{D}_{A}$ and $\mathscr{N}_{A}^{\perp}$.

Proposition 2.2. $A^{-1}$ is closed and dense in $\mathscr{K}^{\prime \prime}$ and $\mathscr{N}_{A^{-1}}$ is closed. Moreover, $\left(A^{-1}\right)^{-1}=A$. Furthermore, if $A$ possesses an inverse, then $A^{-1}$ is the inverse of $A$.

Proposition 2.3. The operator $A^{\prime}$ is the adjoint of the pseudoinverse of $A$ and is also the pseudo-inverse of its adjoint, that is,

$$
A^{\prime}=\left(A^{-1}\right)^{*}=\left(A^{*}\right)^{-1} \text {. }
$$

The operators $A$ and $A^{\prime}$ have the same null spaces. Moreover

$$
\left(A^{\prime}\right)^{\prime}=A \text {. }
$$

Proposition 2.4. Let $A$ be an operator from $\mathscr{X}^{\prime}$ to $\mathscr{C}^{\prime \prime}$. Then $A^{-1}, A^{*}$, and $A^{\prime}$ are operators. The products $A^{*} A, A^{-1} A^{\prime}$ are nonnegative, self-adjoint operators, are pseudo-inverses of each other, and have the same null space as $A$. Similarly, the products $A A^{*}, A^{\prime} A^{-1}$ are nonnegative, self-adjoint operators, are pseudo-inverses of each other, and have the same null space as $A^{*}$.

The preceding representation has been suggested by M. R. Hestenes, and the propositions above can be found in the reference [3]. For additional work using these concepts, the reference [1] may be consulted.

The Closed Graph Theorem immediately leads us to the following results: 
THEOREm 2.1. Let $A$ be an operator from $\mathscr{Y}$ to $\mathscr{X}^{\prime}$ Then the range $\mathscr{R}_{A}$ of $A$ is closed if and only if $A^{-1}$ is bounded.

Theorem 2.2. Suppose $A$ and $B$ are operators from $\mathscr{C}$ to $\mathscr{X}^{\prime}$. Suppose $\mathscr{D}_{A} \subseteq \mathscr{D}_{B}$ and $\mathscr{N}_{A} \subseteq \mathscr{N}_{B}$. Suppose further that $A^{-1}$ is bounded. Then $C \equiv B A^{-1}$ is bounded and $\|B x\| \leqq\|C\|\|A x\|$ on $\mathscr{D}_{\mathbf{A}}$.

3. Theorems about an operator $A$ and its *-reciprocal $A^{\prime}$. In this section we are concerned with theorems which connect an operator $A$ and its *-reciprocal $A^{\prime}$. Some of the theorems will be results which relate different norms to each other, i.e., theorems regarding the equivalence of topologies. We also characterize the domain of the operator $A^{\prime}$ in terms of the operator $A$.

We begin with the following lemma, which is a "generalized" Schwarz inequality similar to that used by P. Lax in [5] and M. Schechter in [6]. This will prove useful later on.

Let $A$ be an operator from $\mathscr{H}$ to $\mathscr{H}^{\prime}$. We have $\mathscr{D}_{A} \subseteq \mathscr{H}$. Now, $\mathscr{D}_{A^{\prime}}=\mathscr{R}_{A^{*}} \oplus \mathscr{R}_{A^{*}}^{\perp} \subseteq \mathscr{H}$. Denote $\mathscr{D}_{A} \cap \mathscr{N}_{A}^{\perp}$ by $\mathscr{C}_{A}$, the carrier of $A$.

Lemma 3.1. Let $x \in \mathscr{D}_{A}, y \in \mathscr{D}_{A^{\prime}}$, and either $x$ or $y$ in $\mathscr{N}_{A}^{\perp} \equiv$ $\mathscr{N}_{A^{\prime}}^{\perp}$. Then

$$
(x, y)=\left(A x, A^{\prime} y\right)
$$

and

$$
|(x, y)| \leqq\|A x\|\left\|A^{\prime} y\right\| \text {. }
$$

The relation (3.1) follows immediately by observing that

$$
(x, y)=\left(A^{-1} A x, y\right)=\left(A x, A^{\prime} y\right) .
$$

Equation (3.1) can be used to define the domain of the operator $A^{\prime}$ as those vectors $y$ for which there is a vector $z \in \overline{\mathscr{R}_{A}}$ such that $(x, y)=$ $(A x, z)$ for all $x$ in $\mathscr{C}_{A}$.

Equation (3.2) follows directly from equation (3.1) by use of the Schwarz inequality.

The following theorem is a characterization of the domain of $A^{\prime}$.

THeOREM 3.1. Let $A$ be an operator from $\mathscr{H}$ to $\mathscr{H}^{\prime}$. Then the domain of $A^{\prime}$ is the set $S$ of vectors $y$ for which there is a positive constant $k$ such that

$$
|(x, y)| \leqq k\|A x\|
$$


for all $x$ in the carrier $\mathscr{C}_{\boldsymbol{A}}$ of $A$.

In order to prove this, let $y$ be in $\mathscr{D}_{\mathbf{A}^{\prime}}$ and consider the relation

$$
|(x, y)|=\left|\left(A x, A^{\prime} y\right)\right| \leqq\|A x\|\left\|A^{\prime} y\right\| \text {. }
$$

It follows that

$$
|(x, y)| \leqq k\|A x\| \text { where } k=\left\|A^{\prime} y\right\| .
$$

Therefore $y \in S$ and

$$
\mathscr{D}_{\mathbf{A}^{\prime}} \subseteq S
$$

Suppose, conversely, that $y \in S$. Consider the linear functional $l(x)=(x, y)$ for all $x$ in $\mathscr{C}_{4}$. We have

$$
|l(x)|=|(x, y)| \leqq k\|A x\|
$$

for every $x \in \mathscr{C}_{A}$ and some constant $k>0$. By (3.6), $l(x)$ is bounded on $\mathscr{C}_{A}$. Let $x=A^{-1} z$ where $z \in \mathscr{D}_{A^{-1}}$. Then from (3.6) we have

$$
\left|\left(A^{-1} z, y\right)\right| \leqq k\|z\| \text {. }
$$

Therefore, by the definition of the adjoint of $A^{-1}$, we get

$$
\left(A^{-1} z, y\right)=\left(z, A^{\prime} y\right) \text {. }
$$

Hence $y$ is in $\mathscr{D}_{\boldsymbol{A}^{\prime}}$ and

$$
S \subseteq \mathscr{D}_{A^{\prime}}
$$

Combining (3.5) and (3.9), we arrive at the theorem.

The following corollary is a variation of a theorem which appears in the reference [5]. The theorem, as it appears in [5], is a Differentiability Theorem which aids in the determination of regularity of solutions of elliptic partial differential equations. We will use these results in $\S 7$ to get strong solutions of elliptic differential equations from weak solutions.

Corollary. Let $A$ be an operator from $\mathscr{Y}$ to $\mathscr{S}^{\prime}$. Suppose $\mathscr{D}$ is a subspace of the domain $\mathscr{D}_{A^{\prime}}$, of $A^{\prime}$ such that the closure of $A^{\prime}$ restricted to $\mathscr{D}$ is $A^{\prime}$. If $x$ is an element of $\mathscr{Y}$ satisfying

$$
|(x, y)| \leqq \text { constant }\left\|A^{\prime} y\right\|
$$

for every $y \in \mathscr{D}$. Then $x \in \mathscr{D}_{A}$.

The proof follows immediately, since the linear functional $l(y)=$ $(x, y)$ can be extended boundedly over all of $\mathscr{D}_{\mathbf{A}^{\prime}}$. By Theorem 3.1, 
$x \in \mathscr{D}_{A}$.

Our next result is a lemma that relates the norm of $A^{\prime}$ to the norm of $A$.

Suppose $A$ is an operator from $\mathscr{C}$ to $\mathscr{H}^{\prime}$. We have $\mathscr{D}_{A} \subseteq \mathscr{X}$ and $\mathscr{D}_{\mathbf{A}^{\prime}} \subseteq \mathscr{H}$. Let $y \in \mathscr{H}$. Then we have the following:

Lemma 3.2. If $\sup |(x, y)| /\|A x\|$ is finite where the supremum is taken over all $x \neq 0$ in $\mathscr{C}_{A}$, then $y$ is in $\mathscr{D}_{\mathbf{A}^{\prime}}$ and

$$
\left\|A^{\prime} y\right\|=\sup \frac{|(x, y)|}{\|A x\|},
$$

where the supremum is taken over all $x \neq 0$ in $\mathscr{C}_{4}$.

It follows from Theorem 3.1 that $y$ is in $\mathscr{D}_{A^{\prime}}$. To obtain (3.10), write $x=A^{-1} z$, where $z \in \mathscr{D}_{\mathbb{A}^{-1}}$. Then

$$
\frac{|(x, y)|}{\|A x\|}=\frac{\left|\left(A^{-1} z, y\right)\right|}{\|z\|}=\frac{\left|\left(z, A^{\prime} y\right)\right|}{\|z\|} .
$$

Taking the supremum over all $x \in \mathscr{C}_{\boldsymbol{4}}$, (3.10) follows from (3.11).

REMARK. Lemma 3.1 follows immediately from (3.10).

In the coming sections the formula (3.10) for $\left\|A^{\prime} y\right\|$ will be very useful in defining negative norms in the theory of partial differential equations. We continue with

Theorem 3.2. Suppose $A$ is an operator from $\mathscr{H}$ to $\mathscr{X}^{\prime}$ and $B$ is an operator from $\mathscr{C}$ to $\mathscr{C}^{\prime \prime}$. Suppose further that $\mathscr{D}_{\mathbf{A}} \subseteq \mathscr{D}_{B}$ and there is a constant $M>0$ such that

$$
0<\|B x\| \leqq M\|A x\|
$$

for all $x \neq 0$ in $\mathscr{D}_{A}$. Then $\mathscr{D}_{B^{\prime}} \subseteq \mathscr{D}_{A^{\prime}}$ and

$$
\left\|A^{\prime} y\right\| \leqq M\left\|B^{\prime} y\right\|
$$

for all $y \neq 0$ in $\mathscr{D}_{B^{\prime}}$.

Proof. From (3.1) we have

$$
|(x, y)|=\left|\left(B x, B^{\prime} y\right)\right| \leqq\|B x\|\left\|B^{\prime} y\right\| .
$$

Combining this result with (3.12), it follows that

$$
|(x, y)| \leqq M\|A x\|\left\|B^{\prime} y\right\|
$$

where $x \in \mathscr{C}_{A}, y \in \mathscr{D}_{B^{\prime}}$. Using Theorem 3.1 with $k=M\left\|B^{\prime} y\right\|$, we 
have $\mathscr{D}_{B^{\prime}} \subseteq \mathscr{D}_{A^{\prime}}$. Furthermore, dividing both sides of (3.15) by $\|A x\|$ yields

$$
\frac{|(x, y)|}{\|A x\|} \leqq M\left\|B^{\prime} y\right\|
$$

for all $x \neq 0$ in $\mathscr{C}_{A}, y \in \mathscr{D}_{B^{\prime}}$. Taking the supremum over all $x \in \mathscr{C}_{A}$ in (3.16) and using Lemma 3.2, we have

$$
\left\|A^{\prime} y\right\| \leqq M\left\|B^{\prime} y\right\|
$$

for $y \in \mathscr{D}_{B^{\prime}}$ and the theorem is established.

REMARK. The above theorem is also valid if the null spaces $\mathscr{N}_{A}$ and $\mathscr{N}_{B}$ are equal.

We are immediately led to the following

Theorem 3.3. Suppose $A$ is an operator from $\mathscr{H}$ to $\mathscr{H}^{\prime}, B$ is an operator from $\mathscr{H}$ to $\mathscr{H}^{\prime \prime}$. Suppose further that $\mathscr{D}_{A}=\mathscr{D}_{B}$ and that there exists positive constants $m, M$ such that

$$
m\|A x\| \leqq\|B x\| \leqq M\|A x\|
$$

for $x \neq 0, x \in \mathscr{D}_{A}=\mathscr{D}_{B}$. Then $\mathscr{D}_{B^{\prime}}=\mathscr{D}_{A^{\prime}}$ and

$$
m\left\|B^{\prime} y\right\| \leqq\left\|A^{\prime} y\right\| \leqq M\left\|B^{\prime} y\right\|
$$

for $y \neq 0, y \in \mathscr{D}_{A^{\prime}}=\mathscr{D}_{B^{\prime}}$.

Proof. This follows immediately from Theorem 3.2 and symmetry.

REMARK. The above theorem states that if $A$ and $B$ are nondegenerate operators from $\mathscr{H}$ to $\mathscr{K}^{\prime}$ and from $\mathscr{C}$ to $\mathscr{K}^{\prime \prime}$ respectively, then if $A$ and $B$ generate the same topology, $A^{\prime}$ and $B^{\prime}$ generate the same topology.

TheOREM 3.4. Let $A$ and $B$ be operators from $\mathscr{H}$ to $\mathscr{H}^{\prime}$ and $\mathscr{H}^{\prime \prime}$ respectively. Suppose that $\mathscr{D} \subseteq \mathscr{D}_{A} \cap \mathscr{D}_{B}$ and let $A_{1}$ and $B_{1}$ be the respective closures of $A$ and $B$ restricted to. $\mathscr{D}$. If there exist positive constants $m, M$ such that

$$
m\|A x\| \leqq\|B x\| \leqq M\|A x\|
$$

for $x \neq 0$ in $\mathscr{D}$. Then $\mathscr{D}_{A_{1}}=\mathscr{D}_{B_{1}}$.

The proof follows immediately from (3.19) and the properties of closure. 
4. Hilbert Spaces associated with the operators $A$ and $A^{\prime}$ and related theorems. In this section we study the Hilbert Spaces $\mathscr{H}_{A}$ and $\mathscr{C}_{A^{\prime}}$ which are the Hilbert Spaces associated with the operators $A$ and $A^{\prime}$ respectively. We then formulate some general theorems which relate the spaces to each other. For simplicity we assume that the null space $\mathscr{N}_{A}$ of the operator $A$ is zero. We proceed as follows:

Let $A$ be an operator that takes $\mathscr{C}$ into $\mathscr{H}^{\prime}$ and complete the domain $\mathscr{D}_{A}$ of $A$ with respect to the norm

$$
\|x\|_{A}=\|A x\|
$$

for every $x$ in the domain $\mathscr{D}_{A}$ of $A$. Designate the Hilbert Space obtained by $\mathscr{H}_{\mathbf{A}}$.

Consider the Hilbert Space $\overline{\mathscr{R}}_{\boldsymbol{A}}$ which is the closure of the range of $A$, the norm being the natural norm in $\mathscr{X}^{\prime}$. Designate the Hilbert Space $\overline{\mathscr{R}}$ by $\tilde{\mathscr{H}}$. With (4.1) as norm in $\mathscr{D}_{A}$, the operator $A$ is a norm-preserving map of $\mathscr{D}_{A}$ onto $\mathscr{R}_{A}$. Define $\widetilde{A}$ to be the linear extension of $A$ to a unitary operator on $\mathscr{H}_{A}$ onto $\tilde{\mathscr{H}}$.

In a like manner, complete $\mathscr{D}_{A^{\prime}}$ with respect to the norm

$$
\|x\|_{A^{\prime}}=\left\|A^{\prime} x\right\|
$$

for every $x$ in the domain $\mathscr{D}_{A^{\prime}}$ of $A^{\prime}$. Designate this Hilbert Space by $\mathscr{\mathscr { C }}_{\mathbf{A}^{\prime}}$. Alternatively, we can complete $\mathscr{D}_{\mathbf{A}^{\prime}}$ with respect to the norm $\sup (x, y) \backslash\|A y\|$ where the supremum is over all $y$ in $\mathscr{D}_{A}$ because of

$$
\left\|A^{\prime} x\right\|=\sup _{y \in \mathscr{D}_{A}} \frac{(x, y)}{\|A y\|} \text {. }
$$

Let $\tilde{A}^{\prime}$ be the unitary operator which maps $\mathscr{\mathscr { C }}_{\mathbf{A}^{\prime}}$ onto $\overline{\mathscr{R}_{\mathbf{A}^{\prime}}}$. Since $\overline{\mathscr{R}_{A}}=\overline{\mathscr{R}_{A^{\prime}}}$, the operator $\widetilde{A}^{\prime}$ maps $\mathscr{H}_{\mathbb{A}^{\prime}}$ onto $\tilde{\mathscr{H}}$. We can now give meaning to the "inner product" of an element in $\mathscr{H}_{A}$ and an element in $\mathscr{H}_{\boldsymbol{A}^{\prime}}$. For, if $x$ is in $\mathscr{H}_{\boldsymbol{A}}, y$ is in $\mathscr{H}_{\boldsymbol{A}^{\prime}}$, define their "inner product" as follows:

$$
\langle x, y\rangle=\left(\widetilde{A} x, \widetilde{A}^{\prime} y\right) .
$$

Clearly, this is well defined and satisfies the requirements for an "inner product". We observe that if $x \in \mathscr{D}_{\boldsymbol{A}}, y \in \mathscr{D}_{\boldsymbol{A}^{\prime}}$, then $\langle x, y\rangle=$ $(x, y)$, the ordinary inner product in $\mathscr{H}$. We immediately obtain the following theorems which are similar to those obtained by P. Lax in [5].

THEOREM 4.1. (Generalized Schwarz Inequality.) If $x \in \mathscr{K}_{\mathbf{A}}$, $y \in \mathscr{X}_{\mathbf{A}^{\prime}}$, then

$$
|\langle x, y\rangle| \leqq\|x\|_{\Delta}\|y\|_{\Lambda^{\prime}} \text {. }
$$


To prove this we use (4.3) and the ordinary Schwarz Inequality:

$$
\begin{aligned}
|\langle x, y\rangle|=\left|\left(\widetilde{A} x, \widetilde{A}^{\prime} y\right)\right| & \leqq\|\widetilde{A} x\|\left\|\widetilde{A}^{\prime} y\right\| \\
& =\|x\|_{\boldsymbol{A}}\|y\|_{\boldsymbol{A}^{\prime}} .
\end{aligned}
$$

The following theorem is a Representation Theorem and can be used in order to obtain existence theory in Elliptic Partial Differential Equations.

THEOREM 4.2. (Representation Theorem.) Every bounded linear functional $l(y)$ over $\mathscr{H}_{A^{\prime}}$ can be represented as

$$
l(y)=\langle x, y\rangle
$$

where $x \in \mathscr{H}_{\mathbf{A}}$.

Proof. Since $\mathscr{H}_{A^{\prime}}$ is a Hilbert Space, we have by the RieszRepresentation Theorem that

$$
l(y)=(z, y)_{\mathscr{C}_{A^{\prime}}}
$$

where $z \in \mathscr{X}_{\boldsymbol{A}^{\prime}}$. By the way we have constructed $\mathscr{C}_{\boldsymbol{A}}$, we can choose $x \in \mathscr{H}_{\boldsymbol{A}}$ such that $\widetilde{A} x=\widetilde{A}^{\prime} z$. Then

$$
(z, y)_{\mathscr{C}_{A^{\prime}}}=\left(\widetilde{A}^{\prime} z, \widetilde{A}^{\prime} y\right)=\left(\widetilde{A} x, \widetilde{A}^{\prime} y\right)=\langle x, y\rangle .
$$

Therefore $l(y)=\langle x, y\rangle$ where $x \in \mathscr{H}_{\Delta}$ and the theorem is complete.

5. An example. In this section we present an example that will serve to illustrate some of the definitions and theorems from preceding sections. Part of the example appears in the reference [3].

Let $\mathscr{H}$ be the class of all real valued Lebesgue square integrable functions $x\left(t_{1}, t_{2}\right)$ on the square $0 \leqq t_{1} \leqq \pi, 0 \leqq t_{2} \leqq \pi$. Then $\mathscr{H}$ is a Hilbert Space over the real numbers with inner product given by

$$
(x, y)=\int_{0}^{\pi} \int_{0}^{\pi} x\left(t_{1}, t_{2}\right) y\left(t_{1}, t_{2}\right) d t_{1} d t_{2} .
$$

Suppose that $\mathscr{A}$ is now the subclass of $\mathscr{H}$ having the following properties:

(i) $x\left(t_{1}, t_{2}\right)$ is absolutely continuous in $t_{1}$ on $0 \leqq t_{1} \leqq \pi$ for almost all $t_{2}$ on $0 \leqq t_{2} \leqq \pi$ and is absolutely continuous in $t_{2}$ on $0 \leqq t_{2} \leqq \pi$ for almost all $t_{1}$ on $0 \leqq t_{1} \leqq \pi$.

(ii) The partial derivatives $x_{t_{1}}$ and $x_{t_{2}}$ which exist almost everywhere are in $\mathscr{\mathscr { C }}$. 
Let $A$ be the gradient operator written $A x=\operatorname{grad} x$ whose domain $\mathscr{D}_{A}$ consists of those $x$ in $\mathscr{A}$ such that $x\left(0, t_{2}\right)=x\left(\pi, t_{2}\right)=0$ for almost all $t_{2}$ on $0 \leqq t_{2} \leqq \pi$ and $x\left(t_{1}, 0\right)=x\left(t_{1}, \pi\right)=0$ for almost all $t_{1}$ on $0 \leqq t_{1} \leqq \pi$. Then $A$ is a closed, dense operator from $\mathscr{H}$ to $\mathscr{H}^{\prime}$ where $\mathscr{H}^{\prime}$ is the Hilbert Space defined by the Cartesian product $\mathscr{H} \times \mathscr{H}$. The adjoint $A^{*}$ of $A$ is given by

$$
A^{*} y=- \text { divergence } y
$$

where divergence $y$ is the closure of the divergence operator defined on all those $y$ in $\mathscr{H}^{\prime}$ of class $C^{\prime}$. Since the ranges of $A$ and $A^{*}$ are closed, $A$ and $A^{*}$ are reciprocally bounded, i.e., $A^{-1}$ and $A^{\prime}$ are bounded. Observe that the operator

$$
A^{*} A=- \text { Laplacian }=-\left[\frac{\partial^{2}}{\partial t_{1}^{2}}+\frac{\partial^{2}}{\partial t_{2}^{2}}\right]=-\Delta .
$$

In order to derive integral representations for $A^{-1}$ and $A^{\prime}$, consider the functions

$$
x_{m n}\left(t_{1}, t_{2}\right)=\frac{2}{\pi} \sin m t_{1} \sin n t_{2}
$$

where $m, n=1,2,3, \cdots$. These functions form a complete orthonormal system in $\mathscr{H}$ and are eigenfunctions of $A^{*} A=-\Delta$. If $x$ is in $\mathscr{H}$, then

$$
x=\sum_{m, n=1}^{\infty} a_{m n} x_{m n} \quad \text { where } \quad a_{m n}=\left(x, x_{m n}\right)
$$

and convergence is in the mean of order two. The vectors $y_{m n}$ in $\mathscr{H}^{\prime}$ whose components are

$$
\begin{aligned}
& y_{m n}^{1}=\frac{2 m}{\pi \sqrt{m^{2}+n^{2}}} \cos m t_{1} \sin n t_{2}, \\
& y_{m n}^{2}=\frac{2 n}{\pi \sqrt{m^{2}+n^{2}}} \sin m t_{1} \cos n t_{2}
\end{aligned}
$$

form a complete orthonormal system in the range $\mathscr{R}_{A}$ of $A$. Therefore every vector $y$ in $\mathscr{H}^{\prime}$ can be written as

$$
y=y_{0}+\sum_{m, n=1}^{\infty} b_{m n} y_{m n}
$$

where $b_{m n}=\left(y, y_{m n}\right)$ and $y_{0}$ is in $\mathscr{R}_{A}^{\perp}$; i.e., $A^{*} y_{0}=0$.

We have 


$$
\begin{aligned}
A x & =\sum_{m, n=1}^{\infty} \lambda_{m n} a_{m n} y_{m n}, & A^{-1} y & =\sum_{m, n=1}^{\infty} \frac{b_{m n}}{\lambda_{m n}} x_{m n} \\
A^{\prime} x & =\sum_{m, n=1}^{\infty} \frac{a_{m n}}{\lambda_{m n}} y_{m n}, & A^{*} y & =\sum_{m, n=1}^{\infty} \lambda_{m n} b_{m n} x_{m n} \\
A^{*} A x & =\sum_{m, n=1}^{\infty} \lambda_{m n}^{2} a_{m n} x_{m n}, & A A^{*} y & =\sum_{m, n=1}^{\infty} \lambda_{m n}^{2} b_{m n} y_{m n} \\
\left(A^{*} A\right)^{-1} x & =\sum_{m, n=1}^{\infty} \frac{a_{m n}}{\lambda_{m n}^{2}} x_{m n}, & \left(A A^{*}\right)^{-1} y & =\sum_{m, n=1}^{\infty} \frac{b_{m n}}{\lambda_{m n}^{2}} y_{m n} .
\end{aligned}
$$

Consider the following function

$$
\begin{aligned}
& G\left(s_{1}, s_{2}, t_{1}, t_{2}\right) \\
& \quad=\frac{4}{\pi^{2}} \sum_{m, n=1}^{\infty} \frac{1}{\left(m^{2}+n^{2}\right)} \sin m s_{1} \sin n s_{2} \sin m t_{1} \sin n t_{2},
\end{aligned}
$$

which converges uniformly and absolutely. This is the Green's function for the negative of the Laplacian. When plausible, we use the condensed notation $s=\left(s_{1}, s_{2}\right), t=\left(t_{1}, t_{2}\right)$ and $(s, t) \equiv\left(s_{1}, s_{2}, t_{1}, t_{2}\right)$. We observe that

$$
G(s, t)=G(t, s) ; \quad G_{s_{i}}(s, t)=G_{t_{i}}(t, s)
$$

for $i=1,2$, whenever the derivatives exist. The derivatives are given by the formulas

$$
\begin{aligned}
G_{s_{1}}(s, t) & =\frac{4}{\pi^{2}} \sum_{m, n=1}^{\infty} \frac{m}{\left(m^{2}+n^{2}\right)} \cos m s_{1} \sin n s_{2} \sin m t_{1} \sin n t_{2} \\
G_{s_{2}}(s, t) & =\frac{4}{\pi^{2}} \sum_{m, n=1}^{\infty} \frac{n}{\left(m^{2}+n^{2}\right)} \sin m s_{1} \cos n s_{2} \sin m t_{1} \sin n t_{2}
\end{aligned}
$$

where the sums are taken in the mean of order two on the interval $0 \leqq s \leqq \pi, 0 \leqq t_{i} \leqq \pi ; i=1,2$. Define

$$
\begin{aligned}
& K_{1}(s, t)=G_{s_{1}}(s, t)=G_{t_{1}}(t, s) \\
& K_{2}(s, t)=G_{s_{2}}(s, t)=G_{t_{2}}(t, s)
\end{aligned}
$$

If $y=\left(y_{1}, y_{2}\right) \in \mathscr{H}^{\prime}$, then by (5.2), (5.6), and (5.10),

$$
A^{-1} y(t)=\int_{0}^{\pi} K_{1}(s, t) y_{1}(s) d s+\int_{0}^{\pi} K_{2}(s, t) y_{2}(s) d s
$$

for almost all $t$ on $0 \leqq t \leqq \pi$. Similarly, if $y(t)=\left(y_{1}(t), y_{2}(t)\right)=$ $A^{\prime} x \in \mathscr{H}^{\prime}$, then 


$$
\begin{aligned}
& y_{1}(t)=\int_{0}^{\pi} K_{1}(t, s) x(s) d s, \\
& y_{2}(t)=\int_{0}^{\pi} K_{2}(t, s) x(s) d s
\end{aligned}
$$

for almost all $t$ on $0 \leqq t \leqq \pi$. Define

$$
H^{i j}(s, t)=\sum_{m, n=1}^{\infty} \frac{1}{m^{2}+n^{2}} y_{m n}^{j}(s) y_{m n}^{i}(t)
$$

where $i, j=1,2$. From (5.4) and (5.13) we have

$$
\begin{aligned}
& H^{11}(s, t)=\frac{4}{\pi^{2}} \sum_{m, n=1}^{\infty} \frac{m^{2}}{\left(m^{2}+n^{2}\right)^{2}} \cos m s_{1} \sin n s_{2} \cos m t_{1} \sin n t_{2} \\
& H^{12}(s, t)=\frac{4}{\pi^{2}} \sum_{m, n=1}^{\infty} \frac{m n}{\left(m^{2}+n^{2}\right)^{2}} \sin m s_{1} \cos n s_{2} \cos m t_{1} \sin n t_{2} \\
& H^{21}(s, t)=\frac{4}{\pi^{2}} \sum_{m, n=1}^{\infty} \frac{m n}{\left(m^{2}+n^{2}\right)^{2}} \cos m s_{1} \sin n s_{2} \sin m t_{1} \cos n t_{2} \\
& H^{22}(s, t)=\frac{4}{\pi^{2}} \sum_{m, n=1}^{\infty} \frac{n^{2}}{\left(m^{2}+n^{2}\right)^{2}} \sin m s_{1} \cos n s_{2} \sin m t_{1} \cos n t_{2}
\end{aligned}
$$

The $H^{i j}(s, t)$ are the Green's functions for the operator $A A^{*}$ given by (5.6). We have for $y(t)=\left(y_{1}(t), y_{2}(t)\right) \in \mathscr{Z}^{\prime}$,

$$
\begin{aligned}
& \left(A A^{*}\right)^{-1} y_{1}(t)=\int_{0}^{\pi} H^{11}(s, t) y_{1}(s) d s+\int_{0}^{\pi} H^{12}(s, t) y_{2}(s) d s \\
& \left(A A^{*}\right)^{-1} y_{2}(t)=\int_{0}^{\pi} H^{21}(s, t) y_{1}(s) d s+\int_{0}^{\pi} H^{22}(s, t) y_{2}(s) d s .
\end{aligned}
$$

We observe that the kernel functions $K_{1}(s, t)$ and $K_{2}(s, t)$ of the operators $A^{-1}$ and $A^{\prime}$ are related to the functions $H^{i j}(s, t)$ as follows:

$$
\begin{aligned}
& G_{s_{i}}(s, t)=K_{i}(s, t)=-H_{t_{1}}^{1 i}(s, t)-H_{t_{2}}^{2 i}(s, t) \\
& G_{t_{i}}(s, t)=K_{i}(t, s)=-H_{s_{1}}^{i 1}(s, t)-H_{s_{2}}^{i 2}(s, t)
\end{aligned}
$$

for $i=1,2$.

The one-dimensional analogue of the above example with $A$ being the differential operator $d / d t$ follows similarly.

6. The operators $G_{k}, G_{k}^{\prime}$ and negative norms. In order to define ellipticity in terms of certain operators in Hilbert Space it is convenient at this time to define a new operator which we denote by $G_{k}$. This operator has been introduced by M. R. Hestenes in his paper [3]. We proceed as follows:

Let $\Omega$ be a bounded, connected, open set in Euclidean $m$-space with boundary $\partial \Omega$. Let $\alpha=\left(\alpha_{1}, \alpha_{2}, \cdots, \alpha_{m}\right)$ where the $\alpha_{i}$ are nonnegative integers and $|\alpha|=\alpha_{1}+\alpha_{2}+\cdots+\alpha_{m}$. Define $\mathscr{L}_{k}^{n}$ to be 
the class of all Lebesgue square integrable complex-valued functions $y_{\alpha}^{j}(t)$ where $t=\left(t_{1}, \cdots, t_{m}\right) \in \Omega ; j=1, \cdots, n ;|\alpha| \leqq k$. The class $\mathscr{L}_{k}^{n}$ with

$$
(y, z)_{\mathscr{S}_{k}^{n}}=\int_{\Omega} y_{\alpha}^{j}(t) \overline{z_{\alpha}^{j}(t)} d t,
$$

where $j \leqq n,|\alpha| \leqq k$ as its inner product forms a Hilbert Space over the field of complex numbers. Henceforth, denote $(y, z)_{\mathscr{E}_{k}^{n}}$ by $(y, z)_{k}$ or, when clear, by $(y, z)$. Furthermore, by $y_{k}(t)$ we will mean all $y_{\alpha}(t)$ such that $|\alpha|=k$.

Define the operator

$$
D_{\alpha}=\frac{\partial^{|\alpha|}}{\partial t_{1}^{\alpha_{1}} \partial t_{2}^{\alpha_{2}} \cdots \partial t_{m}^{\alpha_{m}}}
$$

where again $\alpha=\left(\alpha_{1}, \cdots, \alpha_{m}\right), \alpha_{i}$ are nonnegative integers and $|\alpha|=$ $\alpha_{1}+\cdots+\alpha_{m}$. For $|\alpha|=0$, define $D_{\alpha}=I$, the identity operator.

We now define a closed subspace $\mathscr{D}_{k}^{n}$ of $\mathscr{L}_{k}{ }^{n}$ as follows: Let $\mathscr{D}_{k}^{n}$ be the set of all $x(t)$ of the form $x_{\alpha}^{j}(t)$ in $\mathscr{L}_{k}^{n}$ such that if we set $x^{j}(t)=x_{0}^{j}(t)$, then $x_{\alpha}^{j}(t)=D_{\alpha} x^{j}(t)$.

The subspace $\mathscr{D}_{k}^{n}$ then consists of those functions and all their derivatives up to order $k$ which are in $\mathscr{L}_{k}{ }^{n}$. Hence, a mapping is defined from $\mathscr{L}_{0}^{n}$ to $\mathscr{L}_{k}^{n}$ which maps a function $x_{0}(t)$ into the set consisting of $x_{0}(t)$ and all of its derivatives of order less than or equal to $k$. We call this mapping $G_{k}$. The range $\mathscr{R}_{G_{k}}$ of $G_{k}$ is $\mathscr{D}_{k}^{n}$ and the domain $\mathscr{D}_{G_{k}}$ of $G_{k}$ is the projection of $\mathscr{D}_{k}^{n}$ on $\mathscr{L}_{0}^{n}$. By the way we have defined $G_{k}$, it is clear that $G_{k}$ is a closed and dense linear transformation from $\mathscr{L}_{0}^{n}$ to $\mathscr{L}_{k}{ }^{n}$. The operator $G_{k}$ is one-to-one, i.e., the null space of $G_{k}$ is zero. We note that $G_{0}=I$, the identity mapping.

In recent years several authors have introduced the "negative norms" in order to deal with problems in Elliptic Partial-Differential Equations. See, for example, the references [5] and [6].

P. Lax and M. Schechter have used the following definition for the "negative norms." For $x \in C^{\infty}$,

$$
\|x\|_{-k}=\sup \frac{(x, y)}{\|y\|_{k}}
$$

where the supremum is taken over all $y \in C^{\infty}$. Here $(x, y)$ denotes the ordinary $\mathscr{L}_{2}$-inner product and $\|y\|_{k}$ the Dirichlet norm

$$
\|y\|_{k}^{2}=\sum_{|\alpha| \leqq k} \int_{\Omega}\left|y_{\alpha}(t)\right|^{2} d t
$$

Observe that

$$
\left\|G_{k} y\right\|=\|y\|_{k}
$$


The interesting relation between the "negative norms" and the operator $G_{k}^{\prime}$ is expressed in the following

\section{THEOREM 6.1. $\left\|G_{k}^{\prime} x\right\|=\|x\|_{-k}$.}

The proof follows immediately from Lemma 3.2.

We denote the Hilbert Spaces which are the completions of the $C^{\infty}$-functions under the $G_{k}$ norm and $G_{k}^{\prime}$ norm by $\mathscr{H}_{k}$ and $\mathscr{K}_{-k}$ respectively.

Using the relations that we have derived between $\mathscr{H}_{A}$ and $\mathscr{H}_{\mathbb{A}^{\prime}}$ in $\S 4$ for an arbitrary operator $A$, we observe that for $A=G_{k}$ and $A^{\prime}=G_{k}^{\prime}$, the range of the operator $G_{k}$, which is the subspace $\mathscr{D}_{k}^{n}$ of $\mathscr{L}_{k}{ }^{n}$, is in one-to-one correspondence with the elements of $\mathscr{H}_{k}$ as well as with the elements of $\mathscr{K}_{-k}$. Similarly, the closure of the range of $G_{k}^{\prime}$, which is also $\mathscr{D}_{k}^{n}$, is in one-to-one correspondence with the elements of $\mathscr{H}_{k}$ as well as with the elements of $\mathscr{H}_{-k}$. The norm of the elements in $\mathscr{C}_{k}$ and in $\mathscr{K}_{-k}$ is taken to be the ordinary Dirichlet norm.

Finally, we observe that the elements in $\mathscr{H}_{k}$ are in one-to-one correspondence with the elements in $\mathscr{X}_{-k}$.

7. Another definition of ellipticity. We begin by defining the concept of ellipticity as is done most often in the literature. We do this for systems of equations.

As before, let $\Omega$ be a bounded, connected, open set in Euclidean $m$-space and denote its boundary by $\partial \Omega$. For the present we will make no assumptions on the boundary. Let $t=\left(t_{1}, \cdots, t_{m}\right)$ denote a point in $\Omega$. Suppose $\alpha=\left(\alpha_{1}, \cdots, \alpha_{m}\right)$ where the $\alpha_{i}$ are nonnegative integers and denote by $|\alpha|$ the sum $|\alpha|=\alpha_{1}+\cdots+\alpha_{m}$. Define

$$
D_{\alpha} x=\frac{\partial^{|\alpha|} x}{\partial t_{1}^{\alpha_{1}} \partial t_{2}^{\alpha_{2}} \cdots \partial t_{m}^{\alpha_{m}}} .
$$

Here and elsewhere, unless otherwise indicated, repeated indices will be summed on. Consider the system

$$
\begin{aligned}
& A^{\sigma} x=p_{\alpha}^{\sigma j}(t) D_{\alpha} x^{j}(t) ； \\
& |\alpha| \leqq k ; \\
& j=1, \cdots, n \text {; } \\
& \sigma=1, \cdots, q \text {. }
\end{aligned}
$$

By the principal part of $A^{\sigma} x$ will be meant

$$
P^{o} x=p_{\alpha}^{\sigma j}(t) D_{\alpha} x^{j}(t) ; \quad|\alpha|=k .
$$

$A$ is said to be elliptic if at each point $t \in \Omega$,

$$
p_{\alpha}^{\sigma j}(t) \xi^{\alpha} \eta^{j}=0 ;
$$$$
|\alpha|=k
$$ 
holds for nonnull complex numbers $\eta=\left(\eta^{1}, \cdots, \eta^{n}\right)$ and real numbers $\xi=\left(\xi_{1}, \xi_{2}, \cdots, \xi_{m}\right)$ only in case $\xi=(0,0, \cdots, 0)$ where

$$
\xi^{a}=\xi_{1}^{\alpha_{1}} \xi_{2}^{\alpha_{2}} \cdots \xi_{m}^{\alpha_{m}} \text {. }
$$

An example of the above for a system of $m$ elliptic differential operators with one function $x(t)$ is the following

$$
A x=\text { Gradient } x=\left(\frac{\partial x}{\partial t_{1}}, \frac{\partial x}{\partial t_{2}}, \cdots, \frac{\partial x}{\partial t_{m}}\right) .
$$

Writing (7.5) out in terms of a system, we have

$$
A^{1} x=\frac{\partial x}{\partial t_{1}}, A^{2} x=\frac{\partial x}{\partial t_{2}}, \cdots, A^{m} x=\frac{\partial x}{\partial t_{m}} .
$$

Here

$$
P^{i} x=A^{i} x
$$$$
i=1, \cdots, m \text {. }
$$

Now, the characteristic polynomials are $\xi_{1}, \cdots, \xi_{m}$ and the condition (7.4) implies that $\xi_{1}=\xi_{2}=\cdots=\xi_{m}=0$, i.e., $\xi=(0, \cdots, 0)$. We observe here that when one function $x(t)$ is involved, it is unnecessary to introduce the $\eta=\left(\eta^{1}, \cdots, \eta^{n}\right)$.

We now wish to define ellipticity in a different way than it was defined above. We then proceed to explain the relationship between this definition and the other. The following definition is advantageous in the sense that it deals with the properties of operators in Hilbert Space. Cf. [3], page 1355. We begin as follows:

Let $C$ be a bounded operator from $\mathscr{D}_{k}^{n}(k>0)$ to a Hilbert Space $\mathscr{L}_{0}^{q}$. Suppose that $B_{k}$ is a closed and dense "restriction" of $G_{k}$ in $\mathscr{H}=\mathscr{L}_{0}^{n}$. The product $A_{k} \equiv C B_{k}$ defines a dense linear transformation. We will say that $A_{k}$ is an elliptic operator of order $k$ if $A_{k}$ is closed.

An example of a "restriction" of $G_{k}$ is the following: Suppose $B_{k}$ is defined to be $G_{k}$ operating on the closure of the subclass of $\mathscr{L}_{k}^{n}$, whose elements are continuous and have $x_{\alpha}^{j}(t)=0,|\alpha|<k$ on the boundary of $\Omega$. We will see at the end of this section how this particular "restriction" is in fact one which is essential in order to relate this definition of ellipticity to the classical one.

Another example of $B_{k}$ is the "restriction" of $G_{k}$ to all those functions which can be extended to be periodic functions on a given period-parallelogram. We will have occasion to use this in Theorem 7.3.

The next theorem characterizes elliptic operators and can be used as an equivalent definition for them. Cf. [3], page 1356. 
THEOREM 7.1. If $A_{k}$ is a differential operator of order $k$ such that

$$
\left\|B_{k} x\right\| \leqq h_{1}\left[\left\|A_{k} x\right\|+\|x\|\right] \leqq h_{2}\left\|B_{k} x\right\|
$$

for constants $h_{1}, h_{2}>0$. Then there exists a bounded operator $C$ such that $A_{k}=C B_{k}$ and $A_{k}$ is closed. Conversely, if $A_{k}=C B_{k}$ is closed, then (7.8) is valid for every $x$ in the domain $\mathscr{D}_{B_{k}}$ of $B_{k}$.

Proof. By (7.8), $\mathscr{D}_{B_{k}} \subseteq \mathscr{D}_{\Delta_{k}}$ and $\mathscr{N}_{B_{k}} \subseteq \mathscr{N}_{A_{k}}$. The operator $B_{k}^{-1}$ is bounded, since $G_{k}^{-1}$ is. By Theorem 2.2, the operator $A_{k} B_{k}^{-1}$ is bounded. Set $C=A_{k} B_{k}^{-1}$. Then $C B_{k} \equiv A_{k} B_{k}^{-1} B_{k} \equiv A_{k}$. We now show that $A_{k}=C B_{k}$ is closed.

Let $x_{n} \in \mathscr{D}_{\Delta_{k}}$,

$$
x_{n} \Rightarrow x, \quad A_{k} x_{n} \Rightarrow y .
$$

Then

$$
\left\|x_{n}-x_{m}\right\| \rightarrow 0 \text { and }\left\|A_{k}\left(x_{n}-x_{m}\right)\right\| \rightarrow 0
$$

as $m, n \rightarrow \infty$. By (7.8),

$$
B_{k}\left(x_{n}-x_{m}\right) \rightarrow 0
$$

as $m, n \rightarrow \infty$. Therefore $B_{k} x_{n}$ converges to some element, say $z$, and $B_{k}$ being closed, implies that

$$
B_{k} x=z \text {. }
$$

And so, $A_{k} x=C B_{k} x=C z$, i.e., $x \in \mathscr{D}_{A_{k}}$. Furthermore, $A_{k} x_{n}=C B_{k} x_{n} \Rightarrow$ $C z$ and from (7.9) we have

$$
y=C z
$$

Therefore, $A_{k}$ is closed.

Conversely, suppose $A_{k} \equiv C B_{k}$ is closed. Consider the mapping of the pairs $\left\{\left(x, B_{k} x\right)\right\}$ in the product-space

$$
I \oplus C:\left\{\left(x, B_{k} x\right)\right\} \rightarrow\left\{\left(x, A_{k} x\right)\right\} .
$$

The operator $I \oplus C$ is a bounded, one-to-one mapping of the subspace $\left\{\left(x, B_{k} x\right)\right\}$ onto the subspace $\left\{\left(x, A_{k} x\right)\right\}$. Since $A_{k}$ and $B_{k}$ are closed, $\left\{\left(x, A_{k} x\right)\right\}$ and $\left\{\left(x, B_{k} x\right)\right\}$ are complete metric spaces. By the OpenMapping Theorem, the inverse of $I \oplus C$ exists and is continuous, i.e., there exists a constant $h_{1}>0$ such that

$$
\left\|(I \oplus C !)^{-1}\left\{\left(x, A_{k} x\right)\right\}\right\| \leqq h_{1}\left\|\left\{\left(x, A_{k} x\right)\right\}\right\| \text {. }
$$

Therefore 


$$
\left\|B_{k} x\right\| \leqq\|x\|+\left\|B_{k} x\right\| \leqq h_{1}\left[\|x\|+\left\|A_{k} x\right\|\right] .
$$

Furthermore,

$$
h_{1}\left[\|x\|+\left\|A_{k} x\right\|\right]=h_{1}\left[\|x\|+\left\|C B_{k} x\right\|\right] \leqq h_{2}\left\|B_{k} x\right\|
$$

where $h_{2}=h_{1}(1+\|C\|)$.

Combining (7.16) and (7.17), we obtain (7.8).

An immediate consequence of Theorem 7.1 is the following:

COROLlaRY. If the null space of the elliptic operator $A_{k}$ is zero, then $A_{k}$ and $B_{k}$ generate the same topology.

Proof. Since the null space of $A_{k}$ is zero, the first half of the inequality (7.8) is equivalent to the statement

$$
\left\|B_{k} x\right\| \leqq h_{3}\left\|A_{k} x\right\|
$$

for some constant $h_{3}$ greater than zero. Furthermore, since $A_{k}=C B_{k}$ where $C$ is bounded, we have

$$
\left\|A_{k} x\right\|=\left\|C B_{k} x\right\| \leqq h_{4}\left\|B_{k} x\right\|
$$

where $h_{4}=\|C\|$ is greater than zero. Combining (7.18) and (7.19), we obtain

$$
\left\|B_{k} x\right\| \leqq h_{3}\left\|A_{k} x\right\| \leqq h_{3} h_{4}\left\|B_{k} x\right\| \text {. }
$$

The above corollary expresses the following important statement, namely, that if $A_{k}$ is an elliptic operator of order $k$ whose null space is zero, then $\left\|A_{k} x\right\|$ and $\left\|B_{k} x\right\|$ are equivalent norms where $B_{k}$ is a closed and dense restriction of $G_{k}$, and so the knowledge of $G_{k}$ relays much about the nature of $A_{k}$.

The following theorem illustrates the relationship between the classical definition of ellipticity and the above definition. It is to be found in the references [3] and [4]. For a proof of this theorem, see [4] by M. R. Hestenes.

Consider the system

$$
A_{k}^{\sigma} x(t)=P^{\sigma} B_{k}^{\sigma} x(t)=p_{\alpha}^{\sigma j}(t) D_{\alpha} x^{j}(t)
$$

where $\sigma=1, \cdots, q ; j=1, \cdots, n ;|\alpha| \leqq k$. Suppose further that the coefficients $p_{\alpha}^{\sigma j}(t)$ are continuous functions of $t$ on the closure of the $m$-dimensional region $\Omega$. Then

THEOREM 7.2. Given a point $t \in \Omega$, suppose there is no nonnull set of real numbers $\xi=\left(\xi_{1}, \cdots, \xi_{m}\right)$ and no nonnull set of complex numbers $\eta=\left(\eta^{1}, \cdots, \eta^{n}\right)$ such that the relations 


$$
p_{\alpha}^{\sigma j}(t) \xi^{\alpha} \eta^{j}=0
$$

where $\sigma=1, \cdots, q ;|\alpha|=k$ holds, and $\xi^{\alpha}=\xi_{1}^{\alpha_{1}} \cdots \xi_{m}^{\alpha_{m}}$. Suppose further that $B_{k}$ is the restriction of $G_{k}$ defined on the closure of the subclass of $\mathscr{L}_{k}^{n}$ which consists of those elements which are continuous and have $D_{\alpha} x^{j}(t)=0,|\alpha|<k$ on the boundary of $\Omega$. Then $A_{k}$ is elliptic (in our sense) and conversely.

Theorem 7.2 states that ellipticity in the classical sense is equivalent to ellipticity in this "new" sense if $B_{k}$ is restricted to those functions whose derivatives up to order $k$ vanish on the boundary of $\Omega$.

An application of the definition of ellipticity is that of gaining strong solutions from "weak solutions" in elliptic partial differential equations. First we need

Definition 7.1. If $A$ is an elliptic operator, then $x$ is said to be a weak solution of $A x=y$ if

$$
\left(x, A^{*} u\right)=(y, u)
$$

for every $u$ in the domain $\mathscr{D}_{\mathbf{A}^{*}}$ of $A^{*}$.

Normally, the set $\{u\}$ is not the whole domain of $A^{*}$ but is such that the closure of the restriction of $A^{*}$ to $\{u\}$ is $A^{*}$ itself. In applications, there usually exists a set of functions of class $C^{\infty}$ of this type.

As an application of some of the preceding results we prove the existence of strong solutions from weak solutions. We assume the underlying domain is a period-parallelogram.

THEOREM 7.3. Let $A_{k}$ be an elliptic operator of order $k$ whose null space is zero. If $x$ is a weak solution of $A_{k} x=y$, then $x$ is in fact a strong solution of $A_{k} x=y$.

Proof. Since $A_{k}$ is elliptic, $A_{k}=C B_{k}$ where $C$ is bounded and $B_{k}$ is the restriction of $G_{k}$ to those functions which can be extended to be periodic functions defined on the period-parallelogram. Clearly, $\mathscr{D}_{A_{k}}=\mathscr{D}_{B_{k}}$ and $\mathscr{D}_{A^{\prime}{ }_{k}}=\mathscr{D}_{B^{\prime}{ }_{k}}$.

Let $v=A_{k}^{*} u$. By $(7.22)$,

$$
\left|\left(x, A_{k}^{*} u\right)\right|=|(x, v)|=|(y, u)|=\left|\left(y, A_{k}^{\prime} v\right)\right| .
$$

By the corollary to Theorem 7.1,

$$
\left\|B_{k} z\right\| \leqq m\left\|A_{k} z\right\| \leqq M\left\|B_{k} z\right\|
$$

for constants $m, M>0$. By Theorem 3.3, we have 


$$
\left\|A_{k}^{\prime} v\right\| \leqq m\left\|B_{k}^{\prime} v\right\| \leqq M\left\|A_{k}^{\prime} v\right\| \text {. }
$$

Using the Schwarz inequality, (7.23), and (7.25), we have:

$$
|(x, v)| \leqq\|y\|\left\|A_{k}^{\prime} v\right\| \leqq m\|y\|\left\|B_{k}^{\prime} v\right\|
$$

for all $v$ of the form $v=A_{k}^{*} u$, that is, for all $v \in \mathscr{D}_{A^{\prime}{ }_{k}} \equiv \mathscr{D}_{B^{\prime} k_{k}}$. By Theorem 3.1, $x \in \mathscr{D}_{B_{k}}$, that is, $x$ is a strong solution of $A_{k} x=y$.

As a more substantial application using the operators $G_{k}$ and $G_{k}^{\prime}$, one can get existence and regularity theory for certain classes of partial differential equations by using the methods of P. Lax [6]. For a treatment of this, see [5].

\section{REFERENCES}

1. A. Ben-Israel and A. Charnes, Contributions to the theory of generalized inverses, J. Soc. Indust. Appl. Math. 11 (1963), 667-699.

2. M. R. Hestenes, Applications of the theory of quadratic forms in Hilbert-Space to the calculus of variations, Pacific J. Math. 1 (1951), 525-581.

3. — Relative self-adjoint operators in Hilbert-Space, Pacific J. Math. 11 (1961), 4. - Quadratic variational theory and linear elliptic partial differential equations, Trans. Amer. Math. Soc. 101 (1961), 306-350.

5. E. M. Landesman, Hilbert-Space Methods in Elliptic Partial Differential Equations, Ph. D. Dissertation, University of California, Los Angeles, 1965.

6. P. Lax, On Cauchy's problem for hyperbolic equations and the differentiability of solutions of elliptic equations, Comm. Pure Appl. Math. 8 (1955), 615-633.

7. M. Schechter, Negative norms and boundary problems, Ann. of Math. 72 (1960), 581-593.

8. A. E. Taylor, Introduction to Functional Analysis, John Wiley and Sons, Inc., New York, 1963.

Received October 15, 1965. This paper represents a part of the author's doctoral thesis. The author wishes to record his indebtedness to Professor M. R. Hestenes, under whom the work was done. The paper was sponsored in part by the Office of Naval Research, the U. S. Army Research Office (Durham), and the National Science Foundation. Reproduction in whole or in part is permitted for any purpose of the United States Government.

UNIVERSity OF CALIForNia, LOS ANGELES

AND

University of California, Santa Cruz 



\section{PACIFIC JOURNAL OF MATHEMATICS}

\section{EDITORS}

H. SAMELSON

Stanford University

Stanford, California

J. P. JANS

University of Washington

Seattle, Washington 98105
J. DugundJI

University of Southern California

Los Angeles, California 90007

RICHARD ARENS

University of California

Los Angeles, California 90024

\section{ASSOCIATE EDITORS}

E. F. BECKENBACH

B. H. NeumanN

F WolF

K. YOSIDA

\section{SUPPORTING INSTITUTIONS}

UNIVERSITY OF BRITISH COLUMBIA

CALIFORNIA INSTITUTE OF TECHNOLOGY

UNIVERSITY OF CALIFORNIA

MONTANA STATE UNIVERSITY

UNIVERSITY OF NEVADA

NEW MEXICO STATE UNIVERSITY

OREGON STATE UNIVERSITY

UNIVERSITY OF OREGON

OSAKA UNIVERSITY

UNIVERSITY OF SOUTHERN CALIFORNIA
STANFORD UNIVERSITY

UNIVERSITY OF TOKYO

UNIVERSITY OF UTAH

WASHINGTON STATE UNIVERSITY

UNIVERSITY OF WASHINGTON

AMERICAN MATHEMATICAL SOCIETY CHEVRON RESEARCH CORPORATION TRW SYSTEMS

NAVAL ORDNANCE TEST STATION

Printed in Japan by International Academic Printing Co., Ltd., Tokyo Japan 


\section{Pacific Journal of Mathematics}

\section{Vol. 21, No. $1 \quad$ November, 1967}

Friedrich-Wilhelm Bauer, Der Hurewicz-Satz................... 1

D. W. Dubois, A note on David Harrison's theory of preprimes . ......... 15

Bert E. Fristedt, Sample function behavior of increasing processes with stationary, independent increments ..................... 21

Minoru Hasegawa, On the convergence of resolvents of operators....... 35

Søren Glud Johansen, The descriptive approach to the derivative of a set function with respect to a $\sigma$-lattice ....................... 49

John Frank Charles Kingman, Completely random measures ............ 59

Tilla Weinstein, Surfaces harmonically immersed in $E^{3} \ldots \ldots \ldots \ldots \ldots . . \ldots 9$

Hikosaburo Komatsu, Fractional powers of operators. II. Interpolation spaces ......................................... 89

Edward Milton Landesman, Hilbert-space methods in elliptic partial differential equations ...................................... 113

O. Carruth McGehee, Certain isomorphisms between quotients of a group algebra ........................................ 133

DeWayne Stanley Nymann, Dedekind groups .................. 153

Sidney Charles Port, Hitting times for transient stable processes ......... 161

Ralph Tyrrell Rockafellar, Duality and stability in extremum problems involving convex functions . ............................ 167

Philip C. Tonne, Power-series and Hausdorff matrices . . .............. 189 